\title{
Données utiles à l'interprétation des mesures de carbone 14 en milieu terrestre
}

\author{
S. Roussel-Debet ${ }^{\mathrm{a}}$ \\ IRSN, Pôle RadioProtection - Environnement, Service d'Étude et de Surveillance de la Radioactivité dans l'Environnement, Laboratoire \\ d'Étude Radioécologique en milieux Continental et Marin (PRP-ENV/SESURE/LERCM), Cadarache, 13115 St-Paul-lez-Durance, France.
}

Reçu le 12 mars 2013 - Accepté le 6 juin 2013

\begin{abstract}
Résumé - L'examen des résultats de mesure de ${ }^{14} \mathrm{C}$, notamment dans le cadre de la surveillance environnementale requiert la connaissance de certaines spécificités du comportement du carbone. Les principaux paramètres susceptibles d'influer sur les mécanismes d'incorporation du carbone dans les matrices environnementales et donc sur les niveaux mesurés en ${ }^{14} \mathrm{C}$ sont succinctement rappelés. Des résultats de mesure d'échantillons biologiques ont été acquis par l'IRSN dans des zones non directement influencées par des rejets d'installations nucléaires; ces chroniques permettent de présenter les valeurs du bruit de fond naturel et rémanent, exprimé en activité spécifique $\left(\mathrm{Bq}{ }^{14} \mathrm{C} / \mathrm{kg} \mathrm{C}\right)$, hors de l'influence contemporaine d'un rejet local de ${ }^{14} \mathrm{C}$.
\end{abstract}

Abstract - Useful data for interpreting carbon-14 measurements in the terrestrial environment. Understanding the results of measurements of ${ }^{14} \mathrm{C}$, in particular for environmental monitoring, involves identifying some specifics of the behavior of this element. The main parameters influencing the mechanisms of incorporation of carbon in the environmental matrices and thus the measured levels of ${ }^{14} \mathrm{C}$ are briefly reviewed. Data were acquired by the IRSN for biological samples, in zones not directly influenced by the atmospheric releases of nuclear facilities; this information allows us to present the values of the natural and artificial background, expressed as specific activity $\left(\mathrm{Bq}{ }^{14} \mathrm{C} / \mathrm{kg} \mathrm{C}\right)$, not including the influence of a local release of ${ }^{14} \mathrm{C}$.

Keywords: ${ }^{14} \mathrm{C} /$ terrestrial environment / background levels

\section{Introduction}

Cet article résume les principales données nécessaires à l'interprétation des mesures de ${ }^{14} \mathrm{C}$ en milieu terrestre, en rappelant les paramètres basiques du comportement du carbone à prendre en compte et d'autre part en indiquant quelles sont les valeurs actuelles du bruit de fond et leur évolution.

\section{Rappels sur le comportement du carbone en milieu terrestre}

\subsection{Données générales}

Dans l'environnement, le ${ }^{14} \mathrm{C}$ peut se trouver sous forme de carbone minéral (dioxyde de carbone $\mathrm{CO}_{2}$, méthane $\mathrm{CH}_{4}$, carbonates...) ou de matières organiques et il suit le cycle du carbone stable. En milieu terrestre, les végétaux incorporent par photosynthèse le ${ }^{14} \mathrm{C}$ présent dans le dioxyde de carbone

\footnotetext{
a sylvie.roussel-debet@irsn.fr
}

atmosphérique (l'incorporation par le système racinaire des molécules carbonées présentes dans la solution du sol est négligeable par rapport à la photosynthèse) et les animaux incorporent le ${ }^{14} \mathrm{C}$ par ingestion de la matière organique de leurs aliments. Le carbone devient ainsi, sous forme organique, partie intégrante de tous les organismes vivants. Le carbone organique n'est quasiment pas labile au sein d'un organisme vivant et il ne s'échange pas avec le carbone ambiant.

La teneur en ${ }^{14} \mathrm{C}$ d'une composante de l'environnement peut s'exprimer en termes d'activité spécifique ${ }^{1} \mathrm{du}{ }^{14} \mathrm{C}$ par rapport au carbone organique total, en becquerels de ${ }^{14} \mathrm{C}$ par kilogramme de carbone $(\mathrm{Bq} / \mathrm{kg} \mathrm{C})$. La particularité de l'activité spécifique est que sa valeur est quasiment la même pour toutes les composantes des milieux atmosphérique et terrestre $\left(\mathrm{CO}_{2}\right.$ de l'air, flore, faune et productions agricoles) prélevées au même moment, lorsque l'activité de l'air reste constante en moyenne, ce qui constitue le fondement des modèles de transfert de cet isotope (e.g., Quinault et al., 1998; IAEA,

\footnotetext{
${ }^{1}$ Cette grandeur ne doit pas être confondue avec l'activité spécifique intrinsèque du radio-isotope : 1,6487 $\times 10^{11} \mathrm{~Bq}$ par gramme de ${ }^{14} \mathrm{C}$.
} 
2010). Ainsi, peut-on considérer que la mesure de l'activité spécifique d'un seul type d'échantillon biologique à croissance annuelle, par exemple, des feuilles d'arbre à feuillage caduc, représente correctement le niveau moyen de concentration du ${ }^{14} \mathrm{C}$ des autres composantes du milieu terrestre et du $\mathrm{CO}_{2}$ de l'air, au cours de la période où la matière organique s'est formée.

Les principales caractéristiques du comportement du carbone qui doivent être prises en compte lorsqu'il s'agit d'interpréter les mesures environnementales du ${ }^{14} \mathrm{C}$ sont passées en revue dans les paragraphes qui suivent.

\subsection{Fractionnement isotopique}

Lors de la photosynthèse, se produit un faible fractionnement isotopique du ${ }^{13} \mathrm{C}$ par rapport au ${ }^{12} \mathrm{C}$. Le ${ }^{14} \mathrm{C}$ est deux fois plus fractionné que le ${ }^{13} \mathrm{C}$ et les résultats des mesures du ${ }^{14} \mathrm{C}$ peuvent être normalisés par rapport au ${ }^{13} \mathrm{C}$. La prise en compte du fractionnement $\mathrm{du}{ }^{14} \mathrm{C}$ aboutit à une correction des valeurs mesurées de l'ordre de $0,4 \%$ en règle générale (pour la plupart des végétaux ${ }^{2}$ des zones tempérées ou froides, i.e., végétaux $\mathrm{C} 3$ et les animaux nourris avec des végétaux $\mathrm{C} 3$ ), 1,6\% pour les végétaux $\mathrm{C} 4$ et les animaux nourris avec des végétaux C4 (en France, essentiellement maïs ou sorgho et animaux élevés hors sols comme les volailles et les porcs, s'ils sont nourris au maïs). Ces corrections, inférieures aux incertitudes de mesure et à la variabilité naturelle, ne présentent pas d'intérêt pour l'interprétation des données contemporaines, en milieu terrestre, sauf dans le cadre de recherches, notamment celles qui concernent l'étude de l'origine des matières organiques des sols.

\subsection{Fonctionnement des végétaux et périodes de rejets}

Les feuilles d'arbres ou d'arbustes caducs ainsi que tous les organes aériens et souterrains des végétaux annuels incorporent le ${ }^{14} \mathrm{C}$ atmosphérique durant leur croissance de l'année, entre la période de la reprise de végétation et le moment du prélèvement. Les feuilles de ces végétaux sont généralement formées au printemps, en quelques jours, ce qui fait que l'on peut attribuer à la mesure des échantillons une date de début d'incorporation $\mathrm{du}{ }^{14} \mathrm{C}$ présent dans l'air (début de la période durant laquelle l'activité du végétal rend compte de celle de l'air). Ces feuilles, une fois développées, ne contiennent quasiment pas de ${ }^{14} \mathrm{C}$ présent dans l'environnement avant le début de leur croissance (absence de rémanence). En revanche, le bois des arbres et arbustes conserve l'empreinte des contaminations passées : les cernes (anneaux de croissance annuels) peuvent précisément servir à la datation ou au suivi de rejets importants et prolongés, comme cela a été le cas sur le site contaminé de Ganagobie en Provence (Leprieur et al., 2003; Roussel-Debet, 2009).

${ }^{2}$ Lors de la photosynthèse, les végétaux dits « $\mathrm{C} 3$ » assimilent le carbone en formant un composé à 3 atomes de carbone (acide phosphoglycérique) ; les végétaux dits « $\mathrm{C} 4$ » forment d'abord un composé à 4 atomes de carbone (l'oxaloacétate).
Pour les végétaux persistants, le transfert à partir d'un organe préexistant (tiges ligneuses, bois, système racinaire) ayant été marqué par du ${ }^{14} \mathrm{C}$ au cours des années précédentes, vers un nouvel organe ayant poussé au cours de l'année en cours, est négligeable vis-à-vis de la matière organique nouvellement formée : on considère ainsi que la matière organique, une fois constituée l'année $n$ ne va pas subir de redistribution l'année $n+1$. Le ${ }^{14} \mathrm{C}$ incorporé via la biomasse foliaire va se retrouver, en fin de croissance, avec la même activité spécifique moyenne dans les feuilles et dans les organes de réserve annuels (par exemple, racines tubéreuses ou tubercules). Néanmoins, le renouvellement du feuillage s'étale sur trois à quatre ans et se produit durant les périodes favorables à la végétation (printemps, été s'il n'est pas trop sec, début d'automne s'il est doux) et est ralenti voire stoppé en hiver. L'activité spécifique de l'ensemble du feuillage (feuilles d'âges différents) conserve la signature des activités de l'air durant les quelques années nécessaires au renouvellement des feuilles; ainsi, ces échantillons fournissent des résultats de mesure plus difficiles à interpréter que les précédents, notamment en cas de variations importantes de l'activité de l'air d'une année à l'autre.

Dans le cas d'une augmentation brutale et de durée limitée de l'activité de l'air, la réponse du végétal dépend non seulement de l'importance (activité et durée) de cette variation, mais également de sa date d'occurrence par rapport au stade de développement des plantes exposées et leur vitesse de croissance. À titre d'illustration, la figure 1 représente :

- une courbe typique (Tsoularis et Wallace, 2002) de croissance de la matière végétale, pour un légume-feuille, pris ici comme exemple. Il s'agit en réalité de l'indice de surface foliaire LAI (Leaf Area Index, $\mathrm{m}^{2} / \mathrm{m}^{2}$ ) en pourcent du LAI atteint à maturité. L'hypothèse, implicite est que la quantité de matière sèche - donc de matière organique et donc de carbone - est proportionnelle au LAI ;

- l'activité spécifique du $\mathrm{CO}_{2}$ de l'atmosphère, pour lequel on a considéré un quasi-doublement en une semaine de l'activité en ${ }^{14} \mathrm{C}$;

- l'activité spécifique du végétal qui se trouvait, durant cet épisode, soit en fin de croissance (1), soit en pleine croissance (2).

Pour une augmentation identique de l'activité spécifique de l'air, l'activité spécifique en ${ }^{14} \mathrm{C}$ des végétaux à maturité est plus élevée dans le cas (2) d'une augmentation d'activité de l'air concomitante à une phase de croissance rapide du végétal que dans le cas (1) en fin de croissance (ce serait la même chose pour un épisode qui interviendrait en tout début de croissance). Évidemment, un épisode de rejet intervenant durant la phase de repos végétatif des végétaux (période hivernale) n'aurait pas d'influence notable sur le végétal, de même que toutes les conditions qui ralentissent ou annihilent la photosynthèse (stress hydrique ou thermique, obscurité...) et sont susceptibles d'interférer avec l'incorporation du $\mathrm{CO}_{2}$.

De ce qui précède, on peut conclure que les mesures de ${ }^{14} \mathrm{C}$ dans un végétal ne permettent pas forcément de mettre en évidence, a posteriori, un rejet de courte durée ou de faible importance. 

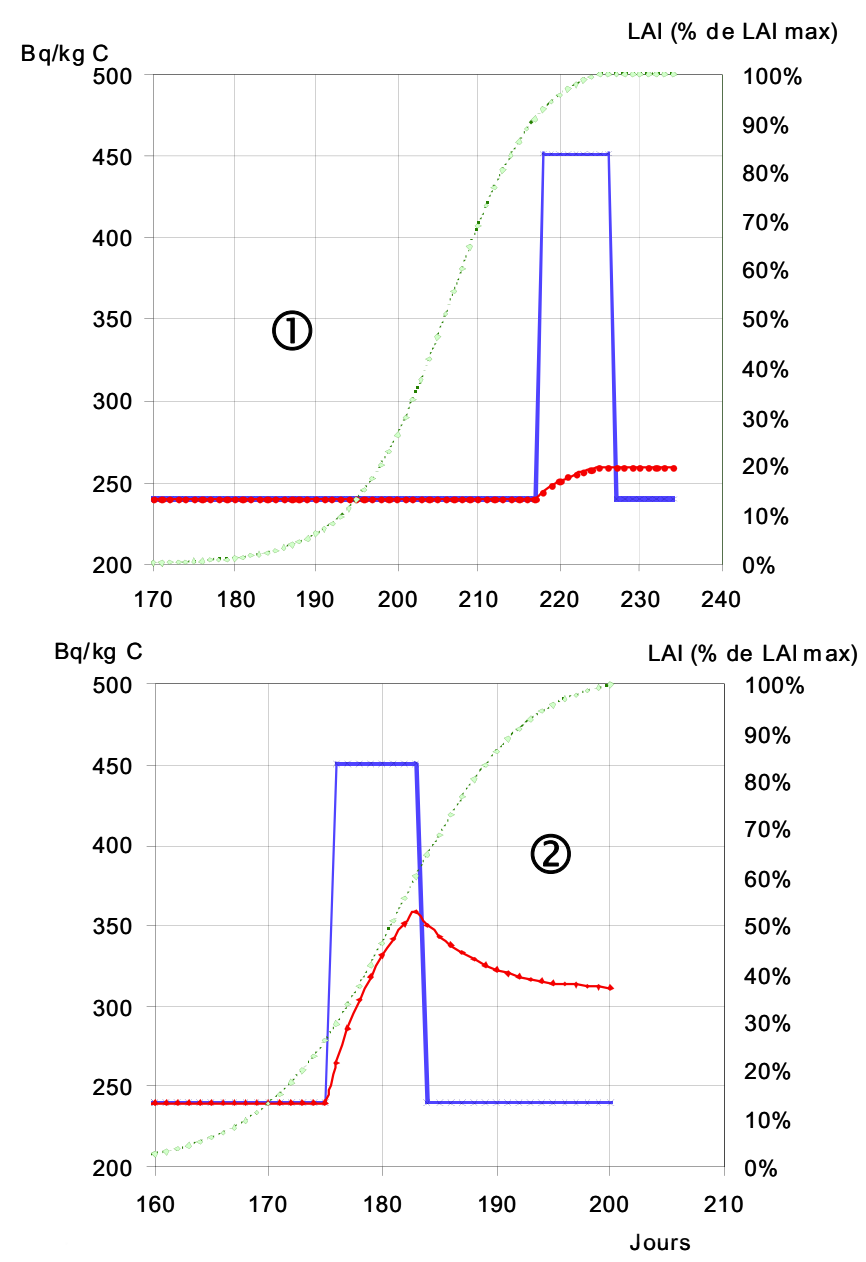

$\longrightarrow$ Activité spécifique du végétal

Doooosoosoos Activité spécifique du $\mathrm{CO}_{2}$ de l'air

...৮.. Accroissement de l'indice foliaire

\section{(1) Végétal en fin de croissance}

\section{(2) Végétal en pleine croissance}

Fig. 1. Illustration d'une courbe de croissance d'un végétal couplée à la variation des activités spécifiques de l'air et du végétal. Illustration of a growth curve for a plant coupled with the variation of the specific activities of the air and of the plant.

\subsection{Comportement du carbone dans le sol}

Dans le sol, lorsque les végétaux meurent, le carbone évolue vers des formes organiques dont la durée de vie varie de quelques mois à plusieurs milliers d'années et il est ré-émis sous forme minérale $\left(\mathrm{CO}_{2}\right)$ dans l'atmosphère. Le ${ }^{14} \mathrm{C}$ émis à partir, d'une part, de la minéralisation de la matière organique des sols et, d'autre part, de la respiration racinaire et microbienne, peut modifier l'activité spécifique du $\mathrm{CO}_{2}$ de la couche d'air qui se trouve au niveau du couvert végétal. La fraction émise peut, en effet, être enrichie ou appauvrie en ${ }^{14} \mathrm{C}$, selon notamment les apports des années précédentes. On considère que cette émanation, qui est à son maximum en été, participerait jusqu'à une valeur de $15 \%$ au carbone total incorporé par photosynthèse, dans le cas de cultures à forte densité de biomasse aérienne, telles que les céréales. Selon que la matière organique du sol est elle-même enrichie ou appauvrie en ${ }^{14} \mathrm{C}$ par rapport à l'air et selon l'intensité de la respiration des racines, cette émanation peut augmenter ou diminuer l'activité spécifique du $\mathrm{CO}_{2}$ utilisé globalement par la plante. Lorsque se produit un enrichissement de l'atmosphère (donc des végétaux) en ${ }^{14} \mathrm{C}$ durant une période suffisamment longue, le résultat des échanges entre les différentes formes du carbone dans le sol, avec des cinétiques variables, se traduit par un accroissement de l'enrichissement du carbone du sol, donc du $\mathrm{CO}_{2}$ émané. L'intensité relative, la cinétique et le retard par rapport au pic initial de ${ }^{14} \mathrm{C}$ atmosphérique de cette réponse varient en fonction de différents facteurs agro-climatiques qui influent sur le temps moyen de renouvellement du carbone du sol (Balesdent, 2002). Sans une connaissance de tout l'écosystème et de son historique, il n'est pas possible de tenir compte de ce biais éventuel. Néanmoins, en sols agricoles, faiblement pourvus en matière organique ( 2 à $4 \%$ ), cette éventuelle contribution d'apports antérieurs est probablement minime. Pour les feuilles d'arbres, cette contribution est négligeable, en raison de la hauteur de la canopée (quelques mètres) par rapport à la hauteur de l'air susceptible d'être affectée par l'émanation (quelques dizaines de centimètres).

\section{Bruit de fond du ${ }^{14} \mathrm{C}$ en milieu terrestre et interprétation}

\section{1 Évolution du bruit de fond du ${ }^{14} \mathrm{C}$ et valeurs récentes acquises en zones non influencées}

La quantité annuelle de ${ }^{14} \mathrm{C}$ formée naturellement est de l'ordre de 1,08 PBq/an et varie suivant différents paramètres cosmiques ; les essais atmosphériques militaires ont injecté environ $250 \mathrm{PBq}$ dans l'atmosphère; les rejets mondiaux actuels de l'industrie nucléaire sont de l'ordre de 0,15 PBq/an (JeanBaptise et Paterne, 2003) dont $7 \%$ pour l'ensemble des rejets gazeux des sites électronucléaires de EDF (Le Guen et Siclet, 2009) et $13 \%$ pour l'usine de retraitement de la Hague (Fontugne et al., 2004). Par ailleurs, depuis les années 1950, la proportion volumique moyenne du $\mathrm{CO}_{2}$ dans l'atmosphère $\left(\mathrm{m}^{3} \mathrm{CO}_{2} / \mathrm{m}^{3}\right.$ air) est passée de $315 \times 10^{-6}$ à plus de $385 \times 10^{-6}$ (en 2009); les données acquises en permanence à l'observatoire international de référence de Mauna Loa, Hawaï, USA (Keeling et al., 2009) reflètent l'augmentation du $\mathrm{CO}_{2}$ atmosphérique, au rythme de 1,9 ppm/an (environ $+3,5 \%$ par an) en moyenne annuelle. Cette moyenne est fluctuante (Povinec et al., 2009) à des échelles différentes : d'une année sur l'autre, ainsi qu'en fonction de la saison (concentration notablement plus élevée en été qu'en hiver (IPCC, 2001)) et à une échelle de temps infra-journalière (Aulagnier et al., 2012; Le Dizès et al., 2012). Outre cette variabilité temporelle, peuvent exister localement des émissions anthropiques liées à la combustion de bois, fuel, essence, gaz naturel... à l'origine de variations significatives de la teneur en $\mathrm{CO}_{2}$ de l'air. La relation 
Tableau 1. Valeurs moyennes annuelles de l'activité spécifique du ${ }^{14} \mathrm{C}$ en milieu terrestre. $n$ : nombre d'échantillons analysés; $\mu$ : moyenne arithmétique; $\sigma$ : écart-type arithmétique.

Mean annual values of the specific activity of ${ }^{14} \mathrm{C}$ in the terrestrial environment. $n$ : number of measured samples; $\mu$ : arithmetic mean; $\sigma:$ standard deviation.

\begin{tabular}{|c|c|c|c|c|}
\hline Année & $n$ & \multicolumn{2}{|c|}{$\mathrm{Bq}{ }^{14} \mathrm{C} / \mathrm{kg} \mathrm{C}$} & \multirow{2}{*}{ Origine des données } \\
\hline & & $\mu$ & $\sigma$ & \\
\hline 2011 & 7 & 232,0 & 1,84 & \multirow{5}{*}{ Cette étude } \\
\hline 2010 & 12 & 233,2 & 1,79 & \\
\hline 2009 & 9 & 238,3 & 3,78 & \\
\hline 2008 & 19 & 236,4 & 5,61 & \\
\hline 2007 & 4 & 241,8 & 0,96 & \\
\hline 2003 & 20 & 242,4 & 2,38 & \multirow{9}{*}{ Roussel-Debet et al., 2006} \\
\hline 2002 & 20 & 246,5 & 3,83 & \\
\hline 2001 & 24 & 248,7 & 3,68 & \\
\hline 2000 & 20 & 247,7 & 2,60 & \\
\hline 1999 & 19 & 250,6 & 3,28 & \\
\hline 1998 & 6 & 251,7 & 5,98 & \\
\hline 1997 & 6 & 252,8 & 6,64 & \\
\hline 1995 & 2 & 256,9 & 4,83 & \\
\hline 1994 & 2 & 257,0 & 1,41 & \\
\hline
\end{tabular}

entre l'augmentation du $\mathrm{CO}_{2}$ de l'air et la diminution de l'activité spécifique du ${ }^{14} \mathrm{C}$ (environ $-0,55 \%$ par an) n'est pas une simple proportionnalité. D'une part, l'augmentation du $\mathrm{CO}_{2}$ de l'air tend à diluer le ${ }^{14} \mathrm{C}$ dans le $\mathrm{CO}_{2}$ qui provient en partie ${ }^{3}$ de l'utilisation des combustibles fossiles dépourvus de ${ }^{14} \mathrm{C}$, d'autre part, les temps de mise à l'équilibre du carbone entre l'atmosphère et les autres compartiments varient de quelques années (végétaux et humus labile) à plusieurs siècles (carbonates marins des abysses, matières organiques réfractaires...), ce qui fait que la réponse de la biosphère est complexe.

Dans les matrices environnementales terrestres, hors de l'influence directe d'une installation nucléaire, l'activité spécifique du ${ }^{14} \mathrm{C}$ décroît depuis l'arrêt des essais nucléaires atmosphériques (Roussel-Debet, 2007). L'évolution de l'activité spécifique du ${ }^{14} \mathrm{C}$ atmosphérique, dans l'hémisphère nord, a été déduite de celle des cernes de bois, permettant de reconstruire les activités, a posteriori (McNamara et McCartney, 1998; Isogai et al., 2002; McGee et al., 2004) ou de mesures de diverses matrices dont l'activité spécifique était considérée en équilibre avec le $\mathrm{CO}_{2}$ de l'atmosphère (Otlet et al., 1997). Dans le prolongement de ces études, l'IRSN a entrepris, de 1994 à 2003, d'actualiser la mesure de ce bruit de fond à partir d'échantillons variés (Roussel-Debet et al., 2006).

Depuis 2007 ces données ont été complétées chaque année par des mesures d'échantillons divers - plus particulièrement des feuillages annuels depuis les cinq dernières années - prélevés sur des stations choisies en dehors de l'influence potentielle d'un site nucléaire (Massif Central, Provence, Bretagne...), au minimum à plus de $20 \mathrm{~km}$ d'un point de rejet. Les mesurages ont été faits par synthèse du benzène

\footnotetext{
${ }^{3}$ Environ 1/4 des émissions de $\mathrm{CO}_{2}$ émis dans l'atmosphère provient d'autres sources (i.e., bois de chauffage, incendies de forêts, émanation du sol...) (IPCC, 2001) ; cette fraction contient du ${ }^{14} \mathrm{C}$, en raison de la teneur plus élevée en ${ }^{14} \mathrm{C}$ atmosphérique durant les essais militaires des années 60 , coïncidant plus ou moins avec la période de formation de ces sources carbonées.
}

jusqu'en 2008 puis par AMS (spectromètre de masse par accélérateur). Les activités spécifiques moyennes annuelles de ces échantillons, reportées tableau 1 et figure 2, se placent dans le prolongement de la courbe de décroissance observée précédemment (Roussel-Debet et al., 2006). L'ensemble des données acquises depuis 1994 peut être ajusté, avec un coefficient de détermination $R^{2}=0,9678$ à une exponentielle décroissante, de période apparente 119 ans, selon l'équation : $\boldsymbol{y}=2,953 \times 10^{7} \times \exp \left(-5,842 \times 10^{-3} \boldsymbol{x}\right)$, où $\boldsymbol{y}$ est l'activité spécifique en $\mathrm{Bq}{ }^{14} \mathrm{C} / \mathrm{kg} \mathrm{C}$ et $\boldsymbol{x}$ est l'année. La période apparente évaluée en 2006 était très proche (117 ans), avec un coefficient $R^{2}=0,95$, ce qui confirme la robustesse de cet ajustement.

\section{2 Équivalences entre les activités spécifiques du ${ }^{14} \mathrm{C}$ et la dose efficace}

En raison de la faible énergie de son rayonnement (émetteur $\beta^{-}$d'énergie moyenne $5 \mathrm{keV}$ ), le ${ }^{14} \mathrm{C}$ n'occasionne pas de dose significative par irradiation externe. L'exposition interne par inhalation de carbone sous forme gazeuse (i.e., $\mathrm{CO}_{2}$ ) est largement inférieure à la dose par ingestion : à activité spécifique du carbone équivalente dans le $\mathrm{CO}_{2}$ de l'air et dans le carbone des denrées, l'inhalation induit une dose de l'ordre de deux pour dix mille de la dose par ingestion. En pratique, seule la dose par ingestion est à considérer. L'ingestion de carbone est assez constante ( 250 à $300 \mathrm{~g} /$ jour pour l'adulte), ainsi la connaissance de l'activité spécifique du carbone dans l'environnement terrestre permet-elle une évaluation simplifiée de la dose efficace, en s'affranchissant des hypothèses sur les rations alimentaires. Avec un facteur de dose efficace pour l'adulte égal à $5,8 \times 10^{-10} \mathrm{~Sv} / \mathrm{Bq}$ ingérés, pour les niveaux habituellement rencontrés dans l'environnement et une consommation moyenne de carbone, la dose efficace est de l'ordre de la dizaine de micro-Sieverts par an ; une augmentation de l'activité spécifique du ${ }^{14} \mathrm{C}$ atmosphérique de $20 \mathrm{~Bq} / \mathrm{kg} \mathrm{C}$ augmente la dose efficace de l'ordre de $1 \mu \mathrm{Sv} / \mathrm{an}$.

\section{Conclusion}

Ce bref rappel des connaissances relatives au ${ }^{14} \mathrm{C}$ met en évidence quelques points clefs. En milieu terrestre, les données sont nombreuses et la bibliographie abondante. L'activité spécifique d'un seul type de matrice, par exemple des feuilles annuelles, est un indicateur particulièrement robuste, apte à refléter de façon sensible le niveau moyen $\mathrm{du}{ }^{14} \mathrm{C}$ de l'atmosphère et de l'écosystème terrestre en général (sols exceptés), sur une durée de quelques semaines à plusieurs mois; en revanche, la mesure de l'activité spécifique ne permet pas toujours de déceler a posteriori une augmentation ponctuelle de l'activité en ${ }^{14} \mathrm{C}$ de l'air. Le bruit de fond moyen en métropole est connu et l'évolution de sa valeur moyenne annuelle peut être déterminée empiriquement de façon satisfaisante; elle peut servir d'élément de comparaison approprié lors de l'examen des résultats de mesure obtenus dans l'environnement des sites nucléaires.

Remerciements. Les prélèvements et les prétraitements des échantillons ont été effectués par Gilles Salaun (IRSN/PRP- 


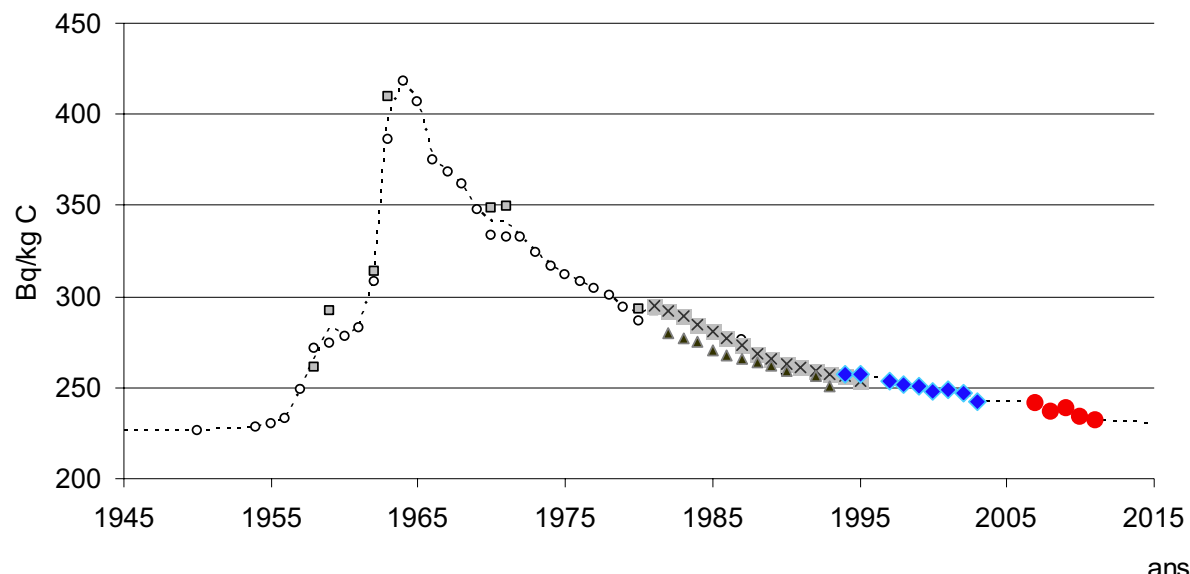

- McGee et al., 2004

口 Isogai et al., 2002

ans

$x$ Otlet et al., 1997

- Roussel-Debet et al., 2006

^ Mc Namara and Mc Cartney, 1998

- Cette étude

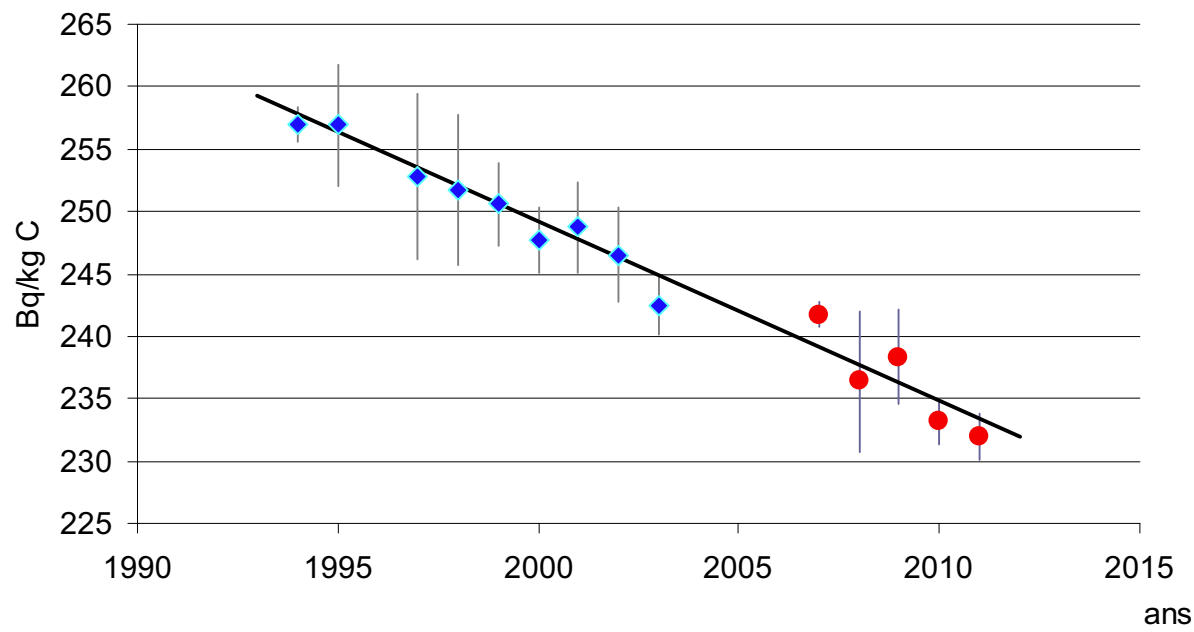

- Roussel-Debet et al., 2006 Cette étude

Fig. 2. Évolution du bruit de fond moyen annuel du ${ }^{14} \mathrm{C}$ en milieu terrestre et ajustement des données acquises récemment. Evolution of the mean annual background activity of ${ }^{14} \mathrm{C}$ in the terrestrial environment and adjustment of recently acquired data.

ENV/SESUR/LERCM). Le Laboratoire de mesure de la radioactivité dans l'environnement (IRSN/PRP-ENV/STEME/LMRE) et le Laboratoire de mesure du ${ }^{14} \mathrm{C}$ (LMC-14, CNRS- INSU) ont réalisé les mesurages $\mathrm{du}{ }^{14} \mathrm{C}$.

\section{Références}

Aulagnier C., Le Dizés S., Maro D., Hubert D., Lardy R., Martin R., Gonze M.A. (2012) Modelling the transfer of ${ }^{14} \mathrm{C}$ from the atmosphere to grass: A case study in a grass field near AREVANC La Hague, J. Environ. Radioact. 112, 52-59.

Balesdent J. (2002) Cycle et temps de résidence du carbone dans les écosystèmes terrestres, approches isotopiques, travaux présentés à la SFRP, Paris, le 18/04/2002.

Fontugne M., Maro D., Baron Y., Hatté C., Hebert D., Douville E. (2004) ${ }^{14} \mathrm{C}$ Sources and Distribution in the Vicinity of La Hague Nuclear Reprocessing Plant: Part I - Terrestrial Environment, Radiocarbon 46 (2), 827-830.
IAEA (2010) Handbook of parameter values for the prediction of radionuclide transfer in terrestrial and freshwater environments. TRS 472. Agence internationale de l'énergie atomique, Vienne.

IPCC (2001) Climate Change 2001 - Scientific basis. The Carbon Cycle and Atmospheric Carbon Dioxide. Contribution of Working Group I to the Third Assessment Report of the Intergovernemental Panel on Climate Change pp. 191-199. Cambridge University press, NY, 881 p.

Isogai K., Cook G.T., Anderson R. (2002) Reconstructing the history of ${ }^{14} \mathrm{C}$ discharges from Sellafield: Part 1 - atmospheric discharges, J. Environ. Radioact. 59 (2), 207-222.

Jean-Baptiste P., Paterne M. (2003) Carbone 14 et environnement global : variabilité naturelle et apports anthropiques, Radioprotection 38 (3), 377-390.

Keeling C.D., Piper S., Bollenbachher A.F., Walker S. (2009) Atmospheric $\mathrm{CO}_{2}$ values (ppm, v) derived from in situ air samples collected at Mauna Loa, Hawaii, USA. Carbon Dioxide Research Group, Institution of Oceanography (SIO), University of California, http://cdiac.ornl.gov/trends/co2/sio-mlo.html. 
Le Dizès S., Maro D., Hébert D., Gonze M.A., Aulagnier C. (2012) TOCATTA : a dynamic transfer model of ${ }^{14} \mathrm{C}$ from the atmosphere to soil-plant systems, J. Environ. Radioact. 105, 48-59.

Le Guen B., Siclet F. (2009) Impact du carbone 14 autour des centrales nucléaires EDF, Radioprotection 44 (4), 495-504.

Leprieur F., Linden G., Pasquier J. (2003) Impact radiologique et sanitaire de la contamination par le carbone 14 du site de Ganagobie (Provence, France), Radioprotection 38 (1), 13-28.

McGee E.J., Gallagher D., Mitchell P.I., Baillie M., Brown D., Keogh S.M. (2004) Recent chronologies for tree rings and terrestrial archives using ${ }^{14} \mathrm{C}$ bomb fallout history, Geochim. Cosmochim. Acta 68 (11), 2509-2516.

McNamara N., McCartney M. (1998) A new estimate of atmospheric ${ }^{14} \mathrm{C}$ discharges from Sellafield, J. Environ. Radioact. 41 (1), 1-10.

Otlet R.L., Walker A.J., Fulker M.J., Collins C. (1997) Background carbon-14 levels in UK foodstuffs, 1981-1995, based upon a 1992 survey, J. Environ. Radioact. 34 (1), 91-101.

Povinec P.P., Holý K., Chudý M., Sivo A., Sýkora I., Jeskovský M., Richtáriková M. (2009) Forty years of atmospheric radiocarbon monitoring around Bohunice nuclear power plant, Slovakia, $J$. Environ. Radioact. 100 (2), 125-130.

Quinault J., Garnier-Laplace J., Roussel-Debet S., Calmon P. (1998) Modélisation des transferts du carbone 14 émis par les réacteurs à eau pressurisée en fonctionnement normal, dans l'environnement proche du site, dans les milieux aquatique d'eau douce et terrestre. Rapport IPSN/SERE 98-07.

Roussel-Debet S. (2007) Evaluation of ${ }^{14} \mathrm{C}$ doses since the end of the 1950s in metropolitan France, Radioprotection 42 (3), 297-313.

Roussel-Debet S. (2009) Évaluations dosimétriques relatives aux options de gestion de l'environnement contaminé en carbone 14 autour de l'ancien laboratoire de la société Isotopchim à Ganagobie. Rapport IRSN/DEI/SESURE/2009-40.

Roussel-Debet S., Gontier G., Siclet F., Fournier M. (2006) Distribution of Carbon-14 in the terrestrial environment close to French nuclear power plants, J. Environ. Radioact. 87, 246-259.

Tsoularis A., Wallace J. (2002) Analysis of logistic growth models, Math. Biosci. 179, 21-55.

Cite this article as: S. Roussel-Debet. Données utiles à l'interprétation des mesures de carbone 14 en milieu terrestre. Radioprotection 49(1), 49-54 (2014). 